\title{
Synthesis of the 5'-phosphonate of 4(S)-(6-amino-9H-purin-9-yl) tetrahydro-2(S)-furanmethanol [S,S-IsoddA]
}

\author{
Vasu Nair* $^{+}$and Pawan K. Sharma ${ }^{+}$ \\ *Department of Pharmaceutical and Biomedical Sciences, R. C. Wilson PH, \\ University of Georgia, Athens, GA 30602 \\ ${ }^{+}$Department of Chemistry, University of Iowa, Iowa City, IA 52242, USA \\ E-mail:vnair@rx.uga.edu
}

(received 19 June 03; accepted 31 July 03; published on the web 07 Aug 03)

\begin{abstract}
4(S)-(6-Amino-9H-purin-9-yl)tetrahydrofuran-2(S)-ylmethyl phosphonic acid, a 5'-Cphosphonate analog of the potent anti-HIV compound $S, S$-IsoddA, was synthesized in order to bypass the critical initial intracellular phosphorylation. Key phases in this multistep synthesis were the Arbuzov reaction of the 5-iodofuranose with triethylphosphite and the Mitsunobu/ coupling reaction of the phosphonate with adenine. The structure of the final product was confirmed by HRMS and multinuclear NMR data.
\end{abstract}

Keywords: Synthesis, isonucleoside, phosphonate, anti-HIV

\section{Introduction}

The unique properties of phosphonate analogs of the natural phosphoric acid esters make them suitable for use in a continuously increasing variety of applications. Replacement of an $O$ -

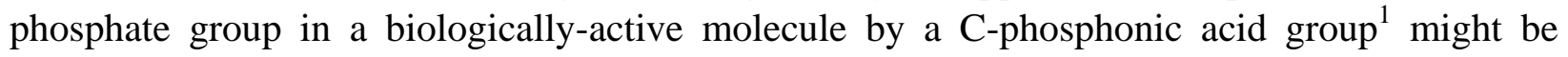
expected to have interesting biological effects. This modification can confer greater stability on these isosteres as the $\mathrm{C}-\mathrm{P}$ bond that replaces the $\mathrm{C}-\mathrm{O}-\mathrm{P}$ bond cannot be hydrolyzed by the enzymes involved in $O$-phosphate ester cleavage. In 1986, an acyclic nucleoside phosphonate analog, (S)-9-(3-hydroxy-2-phosphonomethoxy propyl)adenine, was found to be active against a number of viruses. ${ }^{2}$ Since that time, the use of the apparently membrane permeable phosphonate moiety has been intensively studied in the design of more useful antiviral agents. For example, the anti-HIV activities of dideoxynucleosides are critically dependent on their initial intracellular phosphorylation. ${ }^{3,4}$ One way to overcome the difficulty of the first phosphorylation step is to work with prodrugs that deliver intracellularly the monophosphate forms. ${ }^{5,6}$ Another approach to bypass the first phosphorylation step more completely is through phosphonate analogs, that, after intracellular conversion to their diphosphate forms, can serve as inhibitors/ chain terminators in the HIV RT reaction. ${ }^{7-10}$ A unique feature common to all nucleoside phosphonates is their prolonged antiviral action. 


\section{Results and Discussion}

Nair and coworkers have reported previously on the synthesis ${ }^{11}$ and biological studies ${ }^{12,13}$ of the isomeric dideoxynucleoside, 4(S)-(6-amino-9H-purin-9-yl)tetrahydro-2(S)-furanmethanol, [(S,S)IsoddA] (1). This compound is almost totally resistant to deamination by adenosine deaminase. The triphosphate of $(S, S)$-IsoddA is a potent inhibitor of HIV reverse transcriptase $\left(\mathrm{K}_{i} 16 \mathrm{nM}\right)$. However, the in vitro anti-HIV activity of IsoddA against HIV-1 and HIV-2, although significant, is in the low $\mu \mathrm{M}$ range. Consistent with this is the low level of intracellular phosphorylation of the compound. ${ }^{12}$ In enzymatic studies, we have shown that $(S, S)$-IsoddA is a poor substrate for deoxycytidine kinase, the most likely enzyme for the initial phosphorylation step. ${ }^{14}$ In order to circumvent the critical first phosphorylation step, we have synthesized and studied a phosphatase-stable 5'-C-phosphonate analog of $(S, S)$-IsoddA and the synthesis of this compound is reported here. Only key experimental steps are provided.

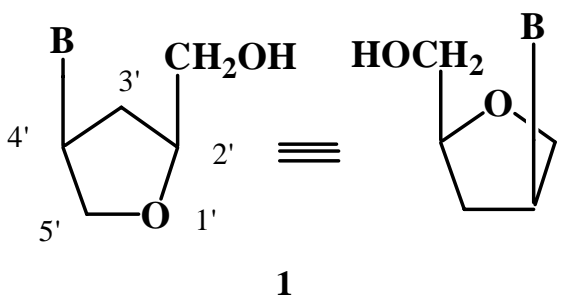

3,5-Dideoxy-5-iodo-1,2-O-isopropylidene- $\alpha$-D-xylo-furanose $(3)^{15}$ was prepared in six steps starting from 1,2-O-isopropylidene- $\alpha-\mathrm{D}$-xylofuranose (2) (Scheme 1). The Arbuzov reaction of 3 with triethylphosphite gave 3,5-dideoxy-1,2-O-isopropylidene-5-diethylphosphonyl- $\alpha$-D-xylofuranose (4) in 85\% yield. Acid-catalyzed methanolysis of 4 afforded the $\alpha$ - and $\beta$-methylglycosides 5 . Reductive demethoxylation of 5 utilized a methodology ${ }^{11}$ which first involved protection of the 2-hydroxyl group by silylation followed in situ, by treatment of this product with triethylsilane and TMS-triflate in 1,2-dichloroethane, which produced the tetrahydrofuran 6 in $46 \%$ yield. Compound 6 can be converted into 4(S)-(6-amino-9H-purin-9-yl)-2(S)diethylphosphonylmethyltetrahydrofuran (7) either under Mitsunobu conditions or by mesylation (89\%) followed by condensation with adenine (49\%). COSY and NOESY NMR data of this compound established unambiguously that only the $\beta$-isomer was obtained during the condensation step. Dealkylation of the phosphonate ester 7 with bromotrimethylsilane gave the corresponding phosphonic acid, 4(S)-(6-amino-9H-purin-9-yl)tetra-hydrofuran-2(S)-ylmethyl phosphonic acid (8) which was purified by preparative HPLC (85\% yield). It was characterized by ${ }^{1} \mathrm{H},{ }^{13} \mathrm{C}$ and ${ }^{31} \mathrm{P}$ NMR data, and high-resolution negative ion mass spectral data. However, evaluation of the compound for in vitro anti-HIV activity in infected CEM-SS cells showed that the activity was much lower than the parent compound, $(S, S)$-IsoddA. 


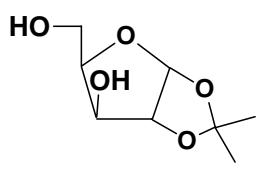

2

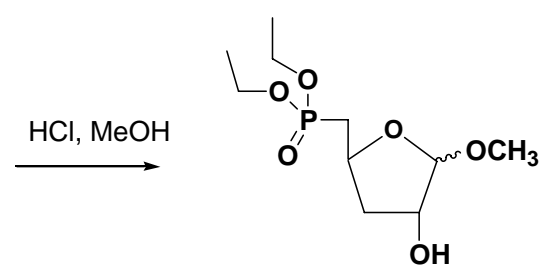

5

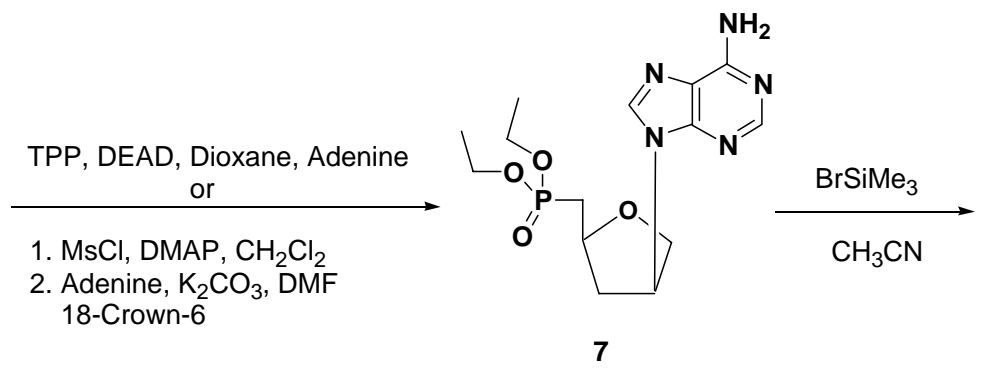

Scheme 1
1. HMDS, TMSCI

2. $\mathrm{Et}_{3} \mathrm{SiH}, \mathrm{TMSOTf}$, $\mathrm{CH}_{2} \mathrm{Cl}_{2}$

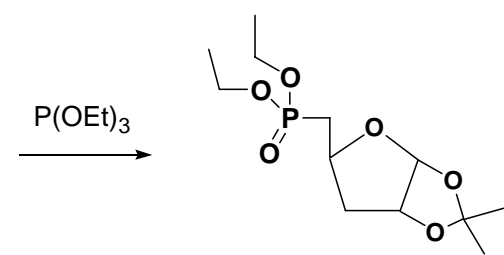

4

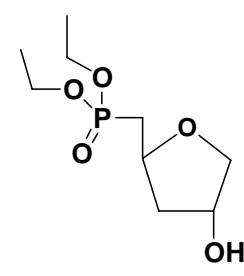

6

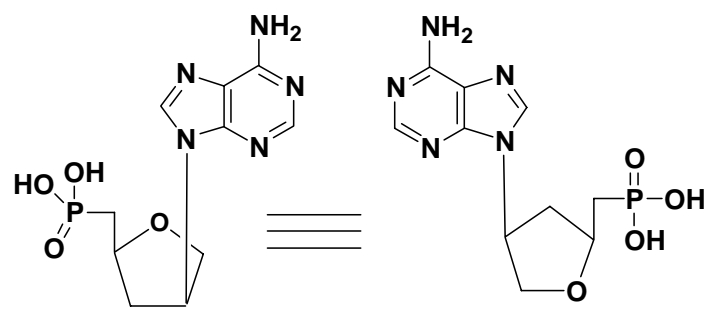

8

\section{Experimental Section}

General Procedures. Nuclear magnetic resonance spectra were recorded on Bruker Model AC300 and WM 360 systems. Ultraviolet spectra were recorded on a Varian Cary Model 3 spectrophotometer. High-resolution FAB mass spectra were obtained on a VG ZAB-HF mass spectrometer. Flash chromatography used 230-400 mesh silica gel. HPLC analyses were carried out on a Beckman-Coulter instrument with C-18 reversed-phase columns.

4(S)-(6-Amino-9H-purin-9-yl)-2(S)-diethylphosphonylmethyltetrahydrofuran (7). A mixture of the mesylate of 6 (178 mg, 0.563 mmole), adenine (152 mg, 1.127 mmole), anhydrous $\mathrm{K}_{2} \mathrm{CO}_{3}$ (156 mg, $1.127 \mathrm{mmole})$, and 18-crown-6 (149 mg, $0.563 \mathrm{mmole})$ in DMF (5 mL) was heated at $75{ }^{\circ} \mathrm{C}$ for $24 \mathrm{~h}$. The solvent was then evaporated under reduced pressure and the residue was stirred with $\mathrm{CHCl}_{3}(250 \mathrm{~mL})$ and filtered. Evaporation of the solvent and column chromatography of the residue under reduced pressure $\left(0\right.$ to $\left.5 \% \mathrm{MeOH} / \mathrm{CHCl}_{3}\right)$ gave pure coupled product 7 (98 mg, 49\%): ${ }^{1} \mathrm{H}$ NMR $\left(\mathrm{CDCl}_{3}\right) \delta 8.32$ (s, $\left.1 \mathrm{H}, \mathrm{H}-2\right), 8.00$ (s, $\left.1 \mathrm{H}, \mathrm{H}-8\right), 5.86$ (br s, $2 \mathrm{H}, \mathrm{NH}_{2}$ ), 5.29 (m, 1H, H-2'), 4.28 (m, 1H, H-4') 4.17 (dd, $1 \mathrm{H}, \mathrm{H}-1^{\prime}, J_{\mathrm{H}^{\prime}-\mathrm{H} 2^{\prime}}=2.4 \mathrm{~Hz}$, $J_{\mathrm{H} 1^{\prime}-\mathrm{H} 1^{\prime \prime}}=10.2 \mathrm{~Hz}$ ), 4.11 and 4.09 (each dq, $2 \mathrm{H}, \mathrm{OCH}_{2} \mathrm{CH}_{3} \times 2, J_{\mathrm{H}-\mathrm{H}}=7.2 \mathrm{~Hz}, J_{\mathrm{H}-\mathrm{P}}=10.8 \mathrm{~Hz}$ ), 4.04 
(dd, 1H, H-1" , $J_{\mathrm{H} 1^{\prime}-\mathrm{H} 1^{\prime \prime}}=10.2 \mathrm{~Hz}, J_{\mathrm{H} 1^{\prime \prime}-\mathrm{H} 2^{\prime}}=6.6 \mathrm{~Hz}$ ), 2.87 (ddd, $1 \mathrm{H}, \mathrm{H}-3^{\prime \prime}, J_{\mathrm{H} 4^{\prime}-\mathrm{H} 3^{\prime \prime}}=7.2 \mathrm{~Hz}, J_{\mathrm{H} 2^{\prime}-}$ $\mathrm{H}^{\prime \prime}=8.4 \mathrm{~Hz}, J_{\mathrm{H}^{\prime}-\mathrm{H} 3^{\prime \prime}}=13.8 \mathrm{~Hz}$ ), 2.37 (ddd, $1 \mathrm{H}, \mathrm{H}-5^{\prime}, J_{\mathrm{H}^{\prime}-\mathrm{H} 4^{\prime}}=5.4 \mathrm{~Hz}, J_{\mathrm{H}^{\prime}-\mathrm{H} 5^{\prime \prime}}=15.0 \mathrm{~Hz}, J_{\mathrm{H}^{\prime}-\mathrm{P}}=18.6$ $\mathrm{Hz}$ ), 2.13 (ddd, 1H, H-5", $J_{\mathrm{H}^{\prime}-\mathrm{H} 5^{\prime \prime}}=7.8 \mathrm{~Hz}, J_{\mathrm{H}^{\prime}-\mathrm{H} 5^{\prime \prime}}=15.0 \mathrm{~Hz}, J_{\mathrm{H}^{\prime}-\mathrm{P}}=18.6 \mathrm{~Hz}$ ) 1.30 and 1.29 (each t, $3 \mathrm{H}, \mathrm{OCH}_{2} \mathrm{CH}_{3} \times 2, \mathrm{~J}=7.2 \mathrm{~Hz}$ ); ${ }^{13} \mathrm{C}$ NMR $\left(\mathrm{CDCl}_{3}\right): \delta 155.75$ (ethylenic C-4 or C-5), $152.83(\mathrm{C}-$ 2), 149.60 (ethylenic C-4 or C-5), 138.04 (C-8), 119.27 (C-6), 74.22 (C-2'), 72.51 (C-5'), 61.79 (m, $\mathrm{P}\left(\mathrm{OCH}_{2} \mathrm{CH}_{3}\right)_{2}$ ), 54.22 (C-4'), 40.03 (d, C-3', J-P $=7.1 \mathrm{~Hz}$ ), 31.95 (d. P-CH, $\mathrm{J}_{\mathrm{C}-\mathrm{P}}=140.5 \mathrm{~Hz}$ ), 16.31 and $16.23\left(\mathrm{P}\left(\mathrm{OCH}_{2} \mathrm{CH}_{3}\right)_{2}\right) ;{ }^{31} \mathrm{P}$ NMR $\left(\mathrm{CDCl}_{3}\right): \delta 26.44(\mathrm{~s})$.

4(S)-(6-Amino-9H-purin-9-yl)tetrahydrofuran-2(S)-ylmethyl phosphonic acid (8). To a solution of 7 (70 mg, 0.197 mmole) in $\mathrm{CH}_{3} \mathrm{CN}$ (3 mL) containing a catalytic amount of dry pyridine $\left(<0.1 \mathrm{~mL}\right.$ ) was added $\mathrm{Me}_{3} \mathrm{SiBr}$ (453 $\mathrm{mg}, 2.96$ mmole) and the reaction mixture was stirred for $96 \mathrm{~h}$ at $25{ }^{\circ} \mathrm{C}$. Pyridine $(0.4 \mathrm{~mL})$ and water $(4 \mathrm{~mL})$ was added and stirring was continued for an additional 2 hours. The reaction mixture was washed with ether $(2 \mathrm{x})$ and the residue was purified by HPLC to give 8 as a crystalline solid (50 mg, 85\%): ${ }^{1} \mathrm{H}$ NMR $\left(\mathrm{D}_{2} \mathrm{O}\right) \delta$ 8.43 and 8.40 (each s, $1 \mathrm{H}, \mathrm{H}-2$ and $\mathrm{H}-8$ ), 5.41 (m, 1H, H-4'), 4.45-4.10 (m, 3H, H-2', H-5' and H-5') 3.05-2.90 (m, 1H, H-3'), 2.35-1.95 (m, 3H, H-3' and $\left.\mathrm{P}^{\prime} \mathrm{CH}_{2}\right) ;{ }^{13} \mathrm{C} \mathrm{NMR}\left(\mathrm{D}_{2} \mathrm{O}\right): \delta 148.77$ (ethylenic C-4 or C-5), 146.18 (C-2), 143.55 (ethylenic C-4 or C-5), 140.16 (C-8), 116.09 (C-6), $73.86\left(\mathrm{C}-2^{\prime}\right), 69.15\left(\mathrm{C}-5^{\prime}\right), 53.59\left(\mathrm{C}-4^{\prime}\right), 36.99$ (d, C-3', J-P $=6.5 \mathrm{~Hz}$ ), 31.75 (d. P-CH${ }_{2}, \mathrm{~J}_{\mathrm{C}-}$ ${ }_{\mathrm{P}}=131.1 \mathrm{~Hz}$ ); ${ }^{31} \mathrm{P}$ NMR $\left(\mathrm{D}_{2} \mathrm{O}\right): \delta 21.92(\mathrm{~s})$; HRMS (ESI) calcd for $\mathrm{C}_{10} \mathrm{H}_{14} \mathrm{~N}_{5} \mathrm{O}_{4} \mathrm{P}:(\mathrm{M}-\mathrm{H})^{-}$ 298.0704, found: 298.0706.

\section{Acknowledgements}

The project described was supported by, Grant Number AI 32851 from the NIH. Its contents are solely the responsibility of the authors and do not necessarily represent the official views of the NIH.

\section{References}

1. (a) Morr, M.; Ernst, L.; Schomburg, D. Liebigs Ann. Chem. 1991, 615. (b) Blackburn, G.M. Chem. Ind. 1981, 134. (c) Engel, R. Chem. Rev. 1977, 77, 349.

2. DeClercq, E.; Holy, A.; Rosenberg, I.; Sakuma, T.; Balzarini, J.; Maudgal, P.C. Nature 1986, 323, 464.

3. (a) Hao, Z.; Cooney, D. A.; Hartman, N. R.; Perno, C-F.; Fridland, A.; DeVico, A. L.; Sarngadharan, M. G.; Broder, S.; Johns, D. G. Mol. Pharmacol. 1988, 34, 431. (b) Cooney, D. A.; Ahluwalia, G.; Mitsuya, H.; Fridland, A.; Johnson, M. A.; Hao, Z.; Dalal, M.; Balzarini, J.; Broder, S.; Johns, D. G. Biochem. Pharmacol. 1987, 36, 1765.

4. De Clercq, E. Nature Reviews: Drug Discovery 2002, 11, 13. 
5. Sastry, J. K.; Nehete, P. N.; Khan, S.; Nowak, B. J.; Plunkett, W.; Arlinghaus, R. B.; Farquhar, D. Mol. Pharmacol. 1992, 41, 441.

6. Puech, F.; Gosselin, G.; Lefebvre, I.; Pompon, A.; Aubertin, A.-M.; Kirn, A.; Imbach, J.-L. Antiviral Res. 1993, 22, 155.

7. Balzarini, J.; Holy, A.; Jindrich, J.; Dvorakova, H.; Hao, Z.; Snoeck, R.; Herdewijn, P.; Johns, D. G.; De Clercq, E. Proc. Natl. Acad. Sci. 1991, 88, 4961.

8. Balzarini, J.; Holy, A.; Jindrich, J.; Naesens, L., Snoeck, R.; Schols, D.; De Clercq, E. Antimicro. Agents Chemther. 1993, 37, 332.

9. Balzarini, J.; Hao, Z.; Herdewijn, P.; Johns, D. G.; De Clercq, E. Proc. Natl. Acad. Sci. 1991, 88, 1499.

10. De Clercq, E. Clin. Microb. Rev. 1997, 10, 674.

11. (a) Nair, V.; Nuesca, Z.M. J. Am. Chem. Soc. 1992, 114, 7951. (b) Bolon, P.J.; Sells, T.B.; Nuesca, Z.M.; Purdy, D.F.; Nair, V. Tetrahedron 1994, 50, 7747.

12. Nair, V.; St. Clair, M.; Reardon, J.E.; Krasny, H.C.; Hazen, R.J.; Paff, M.T.; Boone, L.R.; Tisdale, M.; Najera, I.; Dornsife, R.E.; Everett, D.R.; Borroto-Esoda, K.; Yale, J.L.; Zimmerman, T.P.; Rideout, J. Antimicrob. Agents Chemother. 1995, 39, 1993.

13. Nair, V. In Recent Advances in Nucleosides: Chemistry and Chemotherapy; Chu, C. K., Ed. Elsevier Science: Amsterdam, Netherlands, 2002, p 149.

14. Pal, S.; Nair, V. Biochem. Pharmacol. 2000, 60, 505.

15. Srivastava, V.K.; Lerner, L.M. J. Med. Chem. 1979, 22, 24. 